\title{
Local versus global absorption in thin-film solar cells with randomly textured surfaces
}

C. Rockstuhl, S. Fahr, F. Lederer, K. Bittkau, T. Beckers, and R. Carius

Citation: Appl. Phys. Lett. 93, 061105 (2008);

View online: https://doi.org/10.1063/1.2965117

View Table of Contents: http://aip.scitation.org/toc/apl/93/6

Published by the American Institute of Physics

\section{Articles you may be interested in}

Light localization at randomly textured surfaces for solar-cell applications

Applied Physics Letters 91, 171104 (2007); 10.1063/1.2800374

Light trapping properties of pyramidally textured surfaces

Journal of Applied Physics 62, 243 (1998); 10.1063/1.339189

Light absorption in textured thin film silicon solar cells: A simple scalar scattering approach versus rigorous simulation

Applied Physics Letters 98, 051102 (2011); 10.1063/1.3549175

Absorption enhancement in solar cells by localized plasmon polaritons

Journal of Applied Physics 104, 123102 (2008); 10.1063/1.3037239

The role of oxide interlayers in back reflector configurations for amorphous silicon solar cells Journal of Applied Physics 113, 064508 (2013); 10.1063/1.4790875

Light trapping in solar cells: Analytical modeling

Applied Physics Letters 101, 151105 (2012); 10.1063/1.4758295

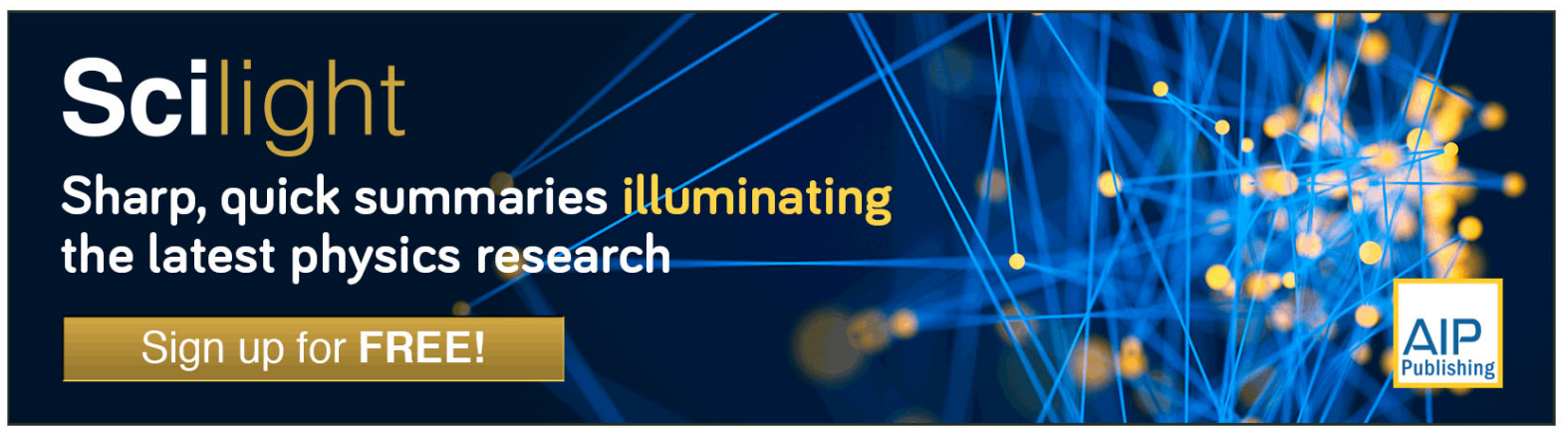




\title{
Local versus global absorption in thin-film solar cells with randomly textured surfaces
}

\author{
C. Rockstuhl, ${ }^{1, \text { a) }}$ S. Fahr, ${ }^{1}$ F. Lederer, ${ }^{1}$ K. Bittkau, ${ }^{2}$ T. Beckers, ${ }^{2}$ and R. Carius ${ }^{2}$ \\ ${ }_{1}^{1}$ Institut für Festkörpertheorie und-optik, Friedrich-Schiller-Universität Jena, Max-Wien Platz 1, 07743 \\ Jena, Germany \\ ${ }^{2}$ Institut für Energieforschung 5, Forschungszentrum Jülich GmbH, 52425 Jülich, Germany
}

(Received 29 May 2008; accepted 7 July 2008; published online 12 August 2008)

\begin{abstract}
Enhanced light absorption in amorphous silicon thin films deposited on randomly textured zinc-oxide surfaces is investigated by means of a rigorous diffraction theory taking into account measured surface profiles and near-field optical data. Global absorption enhancement is obtained in the calculations for particular modifications of the random texture. We furthermore spatially resolve local domains of the surface texture, which show the strongest contribution to the absorption. Criteria on how random surfaces should look like to enhance absorption in thin-film solar cells are derived. (C) 2008 American Institute of Physics. [DOI: 10.1063/1.2965117]
\end{abstract}

Photon management is one of the most promising routes to increase the efficiency of solar cells for a given material. ${ }^{1,2}$ It incorporates all options to modify the optical path and/or the wavelength of light to maximize the generation of electron-hole pairs by absorbed photons in the absorbing layer of solar cells. In this letter, we concentrate on the increase of the optical path length. Simple approaches such as the use of backside reflectors, or antireflection structures, ${ }^{3}$ are already used in large scale production. More sophisticated structures such as subwavelength gratings, ${ }_{5}^{4}$ textured surfaces, or stacks of dielectric layers, ${ }^{5}$ are used in high efficiency solar cells. Even more advanced techniques comprise the incorporation of metallic nanostructures that support surface plasmon polaritons. ${ }^{6}$ Nevertheless, such techniques suffer from the problem that they operate only in a restricted spectral domain and have to be optimized for well defined design wavelengths.

One approach for such kind of photon management that combines most of the aforementioned functionalities and provides a possible solution to the outlined problems is a nonperiodic (i.e., a kind of randomly) textured surface. ${ }^{7} \mathrm{Al}-$ though most of the understanding of random surfaces applies to thick solar cells, it is usually assumed that their functionality is basically twofold, depending on the size of a characteristic feature. (i) For feature sizes much smaller than the wavelength, the surface serves as an antireflector. (ii) For feature sizes much larger than the wavelength, the surface diffuses the light. Although in the indicated limits the explanation is technically sound, reality usually prohibits such simplistic explanations as first, the randomly textured surfaces usually shows feature sizes that are comparable to the wavelength and second, the propagation of light in thin silicon films, having a thickness of only a few hundred nanometers, is not covered by such an analysis. To understand how light absorption is enhanced, particularly in thin-film solar cells, a detailed understanding of light interaction with such surfaces is in need. It has to be the ultimate aim to derive criteria for the definition of preferential surface profiles that maximize absorption in the required wavelength range. ${ }^{8}$ Along this line, recent investigations have already experi-

${ }^{\text {a)} E l e c t r o n i c ~ m a i l: ~ c a r s t e n . r o c k s t u h l @ u n i-j e n a . d e . ~}$ mentally and theoretically revealed light localization effects that occur in close vicinity of randomly textured zinc-oxide $(\mathrm{ZnO})$ surfaces. $^{9}$ Most importantly, it has been shown that light is localized in the near field of such surfaces at locations of high curvature due to the lightning rod effect. In addition, a focusing of light occurs further away in the intermediate field due to an appropriately shaped locally lenslike surface profile. ${ }^{9}$

In this letter, we analyze numerically the absorption enhancement in a thin-film $a$-Si solar cell with a typical thickness. The film is conformally deposited on a random surface. We aim at understanding the effect of a particular surface morphology by modifying it in the simulation and evaluate the respective light trapping efficiency. The analysis is carried out for two wavelengths (658 and $780 \mathrm{~nm}$ ) where efficient light trapping in the solar cell is a mandatory prerequisite to increase the solar cell's efficiency. For this purpose, we use a rigorous diffraction theory to simulate the light propagation in such systems and take into account large scale measured profiles of rough surfaces that are incorporated into real-world solar cells. Complementary to such a global quantity, we will resolve the local light absorption profile to identify spatial domains of the randomly textured surface that provide the largest absorption enhancement.

The textured films used in the present analysis were fabricated by depositing a layer of $1 \mu \mathrm{m} \mathrm{ZnO}$ on glass substrates and a subsequent etching of the films in $0.5 \% \mathrm{HCl}^{10}$ Upon inspection of the surface with an atomic force microscope (AFM), it was found that the depth of the largest craters equals the $\mathrm{ZnO}$ film thickness. A representative surface is shown in Fig. 2(a). From the AFM images, we have chosen a representative surface with a spatial extension of 7 $\times 7 \mu \mathrm{m}^{2}$ that includes all qualitative features hosted by the surface. In the simulation, we assumed that the layer of 250 $\mathrm{nm} a-\mathrm{Si}$ is conformally deposited on the surface.

To determine the absorption, we used the finitedifference time-domain method to calculate at first the local electric field upon illuminating the structure with a plane wave. ${ }^{11}$ Illumination was from the substrate. The dispersive material properties were properly taken into account and values from literature were taken $\left(n_{\mathrm{SiO}_{2}}=1.5, n_{\mathrm{ZnO}}=1.94\right.$, $n_{a-\mathrm{Si}}^{780 \mathrm{~nm}}=3.803+i 0.0022$, and $\left.n_{a-\mathrm{Si}}^{658}{ }^{\mathrm{nm}}=4.074+i 0.046\right) .{ }^{12} \mathrm{To}$ 


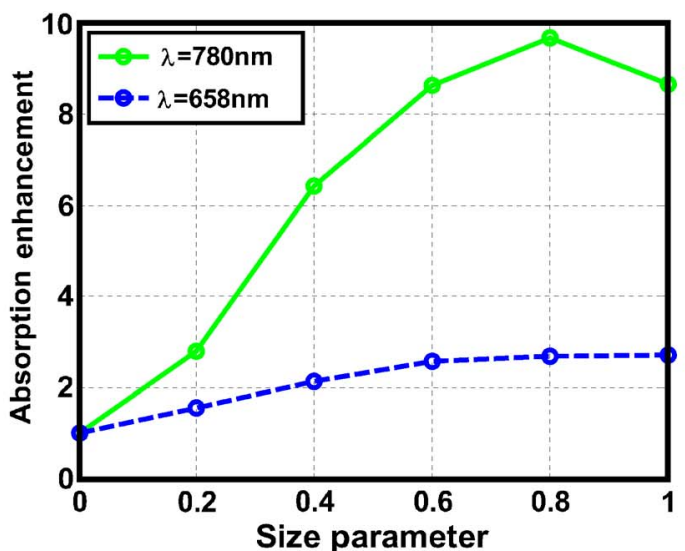

(a)

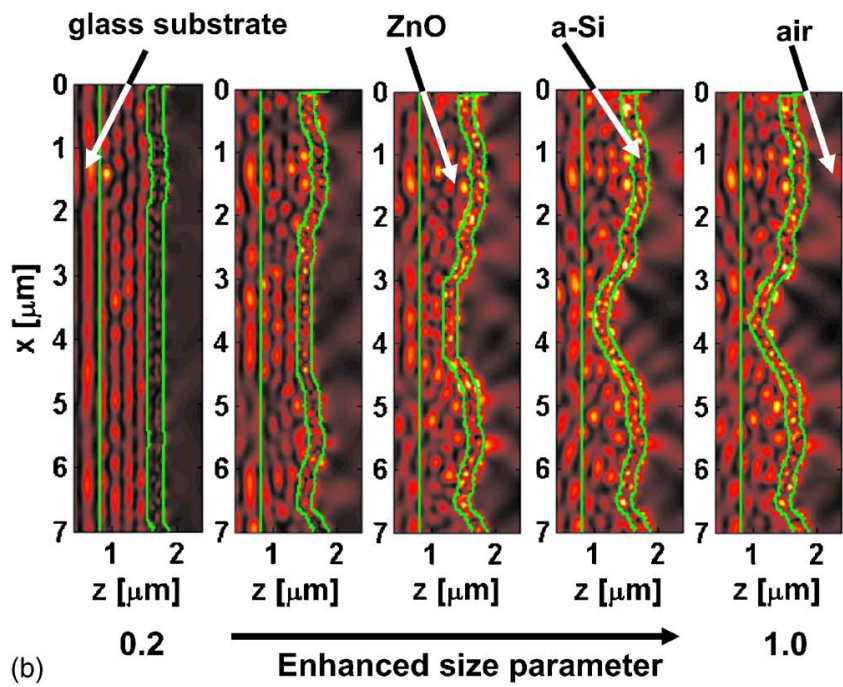

FIG. 1. (Color online) The top figure (a) shows the absorption enhancement at two representative wavelengths in a $250 \mathrm{~nm} a$-Si layer conformally deposited on a rough $\mathrm{ZnO}$ surface as a function of a characteristic size parameter. As deducible from the lower figure (b), the size parameter corresponds to a normalized maximal depth of the rough profile etched into the structure. Lower figures show the light intensity at $780 \mathrm{~nm}$ inside the structure. The green lines indicate the subsequent transition from the quartz substrate, the $\mathrm{ZnO}$ layer, the $a$-Si layer, and finally into the air. The color scale is kept constant in all figures.

truncate the computational window, we have assumed perfectly matched layers in the principal propagation direction ( $z$ direction). Along the $x$ and $y$ axes, periodic boundaries were assumed. We have assumed $x$ polarization for the illumination, as no impact on the results is expected because the structure does not exhibit a preferential symmetry. Once the local electric field is known, the spatially dependent absorption is calculated by the divergence of the Poynting vector $\mathbf{S}$ via $\operatorname{div}\langle\mathbf{S}(\mathbf{r}, \omega)\rangle=-1 / 2 \operatorname{Im}\left\{\omega \mathbf{P}(\mathbf{r}, \omega) \mathbf{E}^{*}(\mathbf{r}, \omega)\right\}$, where $\mathbf{P}$ is the polarization, $\mathbf{E}$ is the electric field, and $\omega$ is the frequency of light. The global absorption is calculated by integrating this absorption over the entire spatial domain of the $a$-Si film. Locally $x$ - and $y$-dependent absorption profiles are calculated by integrating over the $250 \mathrm{~nm}$ in $z$ direction only. All results are normalized with the corresponding absorption in a 250 $\mathrm{nm} a$-Si film deposited on a planar $1 \mu \mathrm{m} \mathrm{ZnO} \mathrm{film.}$

In Fig. 1(a), the changes of the global absorption enhancement by introducing a lower threshold for the depth of the surface profile is shown. In this simulation, all spatial coordinates with a lower depth were clipped to the threshold value, i.e., the deepest craters are filled. For the wavelength
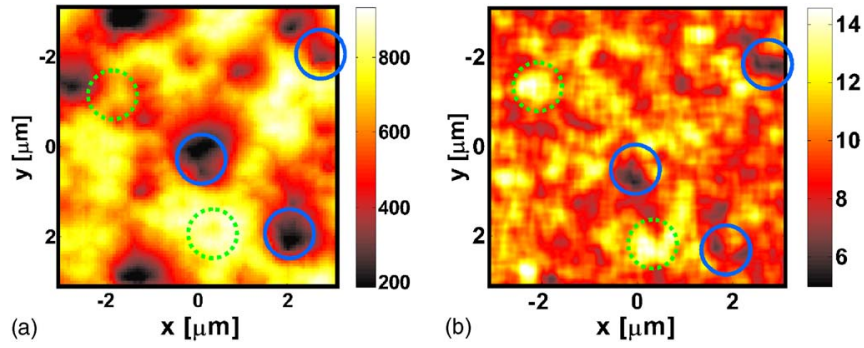

FIG. 2. (Color online) (a) Topography in nanometers of the surface with the modification that causes the highest global enhancement. (b) The local absorption enhancement at a wavelength of $780 \mathrm{~nm}$.

of $780 \mathrm{~nm}$, the absorption is enhanced by a factor of about 9 at full crater depth but up to a factor of 10 if the crater is partially filled (20\% of the full depth). The absorption at the shorter wavelength of $658 \mathrm{~nm}$ is only enhanced up to a factor of 3 . This can be easily explained by considering that the $a$-Si absorption for the flat surface is already high at this wavelength which limits the maximum achievable enhancement. The overall tendency for both curves shows that with increasing depth of the crater the absorption enhancement is stronger. In Fig. 1(b), the local intensity in the central cross section [position $y=0$ in Fig. 2(a)] for different lower threshold values for the depth is shown. The occurrence of hot spots and a generally higher intensity inside the $a$-Si layer is observed. Additional interference patterns can be seen in the $a$-Si film, which result from interference of light propagating inside the $a$-Si layer. Only for rough surface profiles light can couple into the guided modes, causing an enhanced absorption in the $a$-Si layer as demonstrated by the increasing intensity at larger size parameters. From the maximum of the enhancement in Fig. 1(a), one might conclude that either along the rims of the craters the enhancement of absorption is superior and/or in the center of the craters the absorption enhancement is inferior.

To address the important question which spatial domain of the topography contributes how strong to the absorption enhancement, an example of the local absorption profile at a wavelength of $780 \mathrm{~nm}$ along with the associated topography is shown in Fig. 2. The chosen structure corresponds to the surface profile with the largest absorption enhancement, a threshold of $200 \mathrm{~nm}$ for cutting the profile was assumed. The calculated local absorption was spatially averaged within a domain of $280 \mathrm{~nm}$ to reduce the impact of fast oscillations that occur because of interference effects. Comparing the topography (a) and the local absorption profile (b), we observe that our conjecture can be affirmed. In the spatial domain of nearly all crater bottoms the modification causes a weak absorption enhancement. At spatial positions of a local maximum in the topography the absorption is much stronger enhanced as compared to the average enhancement, hence providing a dominant contribution. Circles of different color indicate spatial domains of interest. The light localization that was recently reported on the rims of the craters due to the lightning rod effect seems to be transferred into the $a-\mathrm{Si}^{8}{ }^{8}$ The enhanced local electric field causes a larger dissipation of power in such spatial domains.

To gain further insight into the mechanisms of absorption enhancement, we modified the topology in the simulation in three further ways. In addition to the introduction of a lower threshold for the profile depth shown in Fig. 1, we 


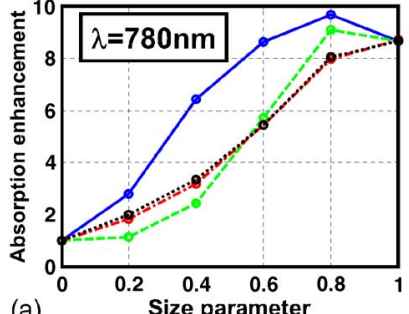

(a)
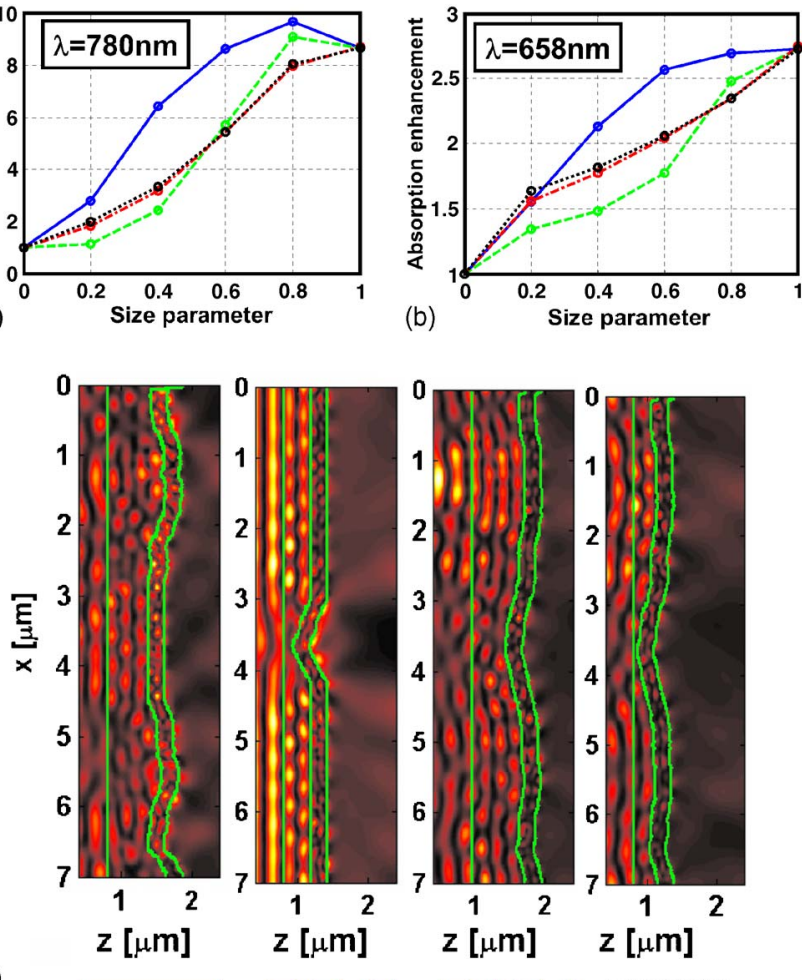

(b)

Size parameter

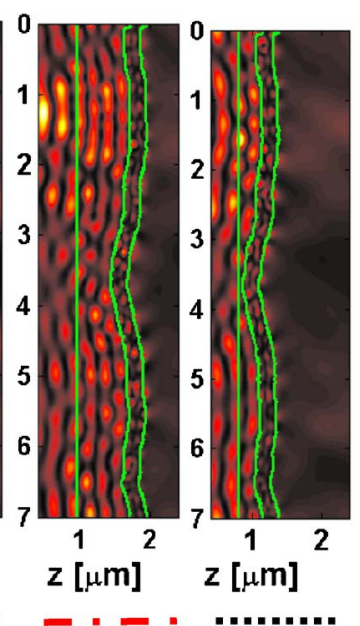

FIG. 3. (Color online) Global absorption enhancement as a function of the normalized strength of a modified topography at a wavelength of $780 \mathrm{~nm}$ (a) and $658 \mathrm{~nm}$ (b). The various means to modify the surface profile are indicated in (c) where the size parameter is always 0.4 . The light intensity there is shown at a wavelength of $780 \mathrm{~nm}$. The solid blue line corresponds to a lower, the green dashed line to an upper threshold of the profile depth. The red dashed-dotted line corresponds to a decrease in the entire magnitude of the surface profile which converges toward a structure where the $a$-Si is deposited on top of a $1 \mu \mathrm{m} \mathrm{ZnO}$ layer. The black dotted line shows the same but the system converges toward a single $a$-Si layer on top of the quartz substrate.

analyzed the impact of an upper threshold for the structure height, e.g., cutting sections of the surface which are above a certain fraction of the maximum thickness $\left(z<z_{\max }\right)$. Finally, we changed the amplitude of the measured surface modulation $z(x, y)$ by the size parameter $(0<s<1)$ by simply scaling the $z$ direction. In such a way we generate a new surface profile $z^{\prime}(x, y)$ that is used in the simulation. This surface function describes the $\mathrm{ZnO}$ interface on which the $a$-Si layer is conformally deposited. At first, the surface profile is modified according to $z^{\prime}(x, y)=(1-s) z_{\max }+s z(x, y)$, i.e., for $s$ $\rightarrow 0$ the surface profile converges toward a $250 \mathrm{~nm}$ thick $a$-Si film on top of a flat $1 \mu \mathrm{m}$ thick $\mathrm{ZnO}$ layer. Second, a surface modification that converges toward a flat $250 \mathrm{~nm}$ thick $a$-Si film on a diminishing $\mathrm{ZnO}$ thickness is considered, i.e., $z^{\prime}(x, y)=s \quad z(x, y)$.

Results for the absorption enhancement in the $a$-Si film as a function of the strength of the surface modification are shown in Fig. 3(a) and 3(b) for the two wavelengths of 780 and $658 \mathrm{~nm}$, respectively. Intensity distributions for the various cases of the surface modification are seen in Fig. 3(c) for a better visualization of the surface modifications.

The results clearly show that the absorption enhancement is weaker if an upper threshold for the profile depth is introduced (green dashed line in Fig. 3) when compared to the introduction of a lower threshold (blue solid line in Fig.
3). The absorption enhancement is also weaker if the surface profile is compressed (dash-dotted red line and dotted black line in Fig. 3, respectively). In particular, the resulting absorption enhancement for the compression of the surface modulation is nearly identical for both scaling variations. This is observed at both wavelengths. No preference for either of the two approaches is encountered. Introducing an upper threshold leads to an even weaker enhancement at small size parameters, i.e., when the surface is almost flat with some pits remaining. For an increased size parameter, the absorption enhancement is larger than for the scaling modification but still weaker than in the case of the lower threshold modification.

The results are in accordance with the explanation given before that the top of the craters does contribute dominantly to the absorption. The introduction of a threshold for these features significantly lowers the absorption. As a general trend for all surface modifications, it can be deduced that the given surface texture exhibits already a very good light trapping behavior, i.e., a high absorption in the $a$-Si layer. Only the introduction of a lower threshold provides a small increase for both wavelengths.

To conclude, we have analyzed the enhancement of absorption in $a$-Si deposited conformally on randomly textured $\mathrm{ZnO}$ surfaces by taking into account surface profiles as used for highly efficient light trapping structures in thin-film silicon solar cells. We discriminated between the local and the global absorption. Overall it was shown that in spatial domains of the surface, where rims and local maxima exist, the absorption enhancement is maximized. By introducing thresholds for the depth of the craters, it was shown that absorption enhancement could be further increased as compared to the prepared samples. Our work gives indications on how the topology of texture etched rough surfaces should preferentially look like for an optimized photon management in thin-film silicon solar cells.

We acknowledge the partial financial support of this work by the Deutsche Forschungsgemeinschaft (PAK88) and the Federal Ministry of Education and Research (Nanovolt). We thank J. Hüpkes for providing us with the samples for this study. Parts of computations were performed on the IBM p690 cluster JUMP of the JvN Forschungszentrum Jülich, Germany and supported by a VSR project.

${ }^{1}$ J. Nelson, The Physics of Solar Cells (Imperial College, London, 2003).

${ }^{2}$ Y. Hamakawa, Thin-Film Solar Cells (Springer, Berlin, 2004).

${ }^{3}$ A. Banerjee and S. Guha, J. Appl. Phys. 69, 1030 (1991)

${ }^{4}$ C. Heine and H. J. Morf, Appl. Opt. 34, 2476 (1995).

${ }^{5}$ J. Zhao, A. Wang, P. Altermatt, and M. A. Green, Appl. Phys. Lett. 66, 3636 (1995).

${ }^{6}$ J. R. Cole and N. J. Halas, Appl. Phys. Lett. 89, 153120 (2006).

${ }^{7}$ E. Yablonovitch and G. D. Cody, IEEE Trans. Electron Devices 29, 300 (1982).

${ }^{8}$ S. Fahr, C. Rockstuhl, and F. Lederer, Appl. Phys. Lett. 92, 171114 (2008).

${ }^{9}$ C. Rockstuhl, F. Lederer, K. Bittkau, and R. Carius, Appl. Phys. Lett. 91 171104 (2007).

${ }^{10}$ O. Kluth, B. Rech, L. Houben, S. Wieder, G. Schöpe, C. Beneking, H Wagner, A. Löffl, and H. W. Schock, Thin Solid Films 351, 247 (1999).

${ }^{11}$ A. Farjadpour, D. Roundy, A. Rodriguez, M. Ibanescu, P. Bermel, J. D. Joannopoulos, S. G. Johnson, and G. Burr, Opt. Lett. 31, 2972 (2006).

${ }^{12}$ O. Vetterl, F. Finger, R. Carius, P. Hapke, L. Houben, O. Kluth, A. Lambertz, A. Mäuck, B. Rech, and H. Wagner, Sol. Energy Mater. Sol. Cells 62, 97 (2000) 